\title{
Effect of PCA Feature Reduction on Ventricular Ectopic Beat Classification
}

\author{
Avvaru Srinivasulu, Y Dileep Kumar
}

\begin{abstract}
Due to the cardiac diseases called Ventricular Tachycardia (VT) and Ventricular Fibrillation (VF) causes sudden cardiac death, it is crucial to recognize Ventricular Ectopic Beats (VEB) in Electrocardio- gram for the early diagnosis. There are many algorithms proposed earlier to classify the VEBs. Even though those algorithms achieved good accu- racy, the size of the feature set is large and not precise. In addition, earlier algorithms used feature reduction methods for reducing the feature set. Therefore, in this paper, we extracted only five features namely, Pre RR interval, post RR interval, $Q R S$ duration, QR slope, and RS slope. Later, we applied the Principle Component Analysis (PCA) for reducing the size of the feature set to observe the effect of Feature Reduction (FR) on the accuracy of VEB Classification. We applied different classifiers for classifying the cardiac beats in to normal and VEBs. Finally, using K-means Nearest Neighborhood (KNN) classifier and cubic Support Vec-tor Machine (SVM) classifier, we achieved $97.4 \%$ classification accuracy, $98.38 \%, 88.89 \%$ \& $98.37 \%$ sensitivity, specificity \& positive predictivity respectively. In addition, it is observed that by applying PCA-FR, the classification accuracy was reduced by a maximum of $3.7 \%$.
\end{abstract}

Keywords: Electrocardiogram (ECG), ventricular ectopic beats (VEB), Feature Reduction (FR), K-means Nearest Neighborhood (KNN) clas- sifier, Support Vector Machine (SVM) classifier, Principle Component Analysis1 (PCA).

\section{INTRODUCTION}

Premature beats originating from ventricular myocardium are called Ventricular Ectopic Beats (VEB) [1]. It was identifiqd.2 as tall and wide beat precede P- wave in ECG [2][3]. The normal and VEB patterns are shown in Figure 1. VEB classification was done previously by extracting many different time domain, frequency domain, statistical and morphological features [4-12]

techniques were applied. But how much degree the FR technique will affect the classification accuracy is not studied. Basically, feature reduction (FR) techniques were used for reducing the feature vector size to reduce the computational time complexity without affecting the actulat 3 accuracy.

To find the most significant features among them, FR As the feature vector size is more, we can achieve good accuracy, so that the researchers will always try to get a greater number of features.

Revised Manuscript Received on December 15, 2019

Avvaru Srinivasulu, Department of Electronics and

Instrumentation Engineering, GITAM, Bangalore campus, India, sreesri.avvaru@gmail.com.

Y Dileep Kumar, Bio-Signal Research Center, Department of Electronics and Instrumentation Engineering, Sree Vidyanikethan Engineering College, Tirupati, India.y.dileepkumar@yahoo.com
But the computational time will be more in such case. Therefore, the feature reduction should be applied in such a way that good accuracy is achieved with smaller feature vector size. In this paper, we have done such an experiment to find the effect of FR on the classification accuracy of VEBs.

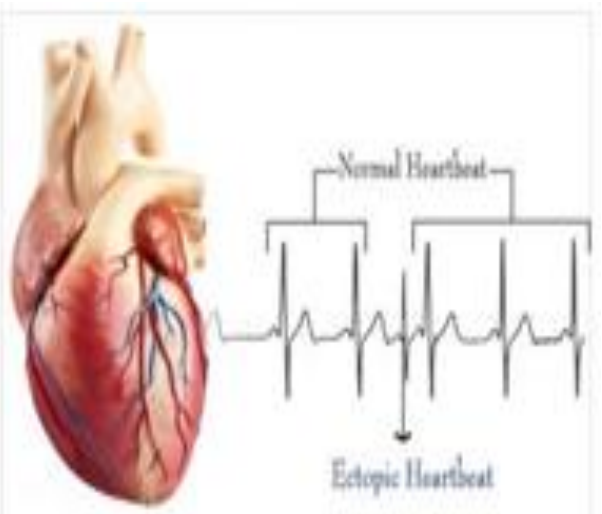

Fig. 1. Normal and Ventricular Ectopic Beats

\section{METHODS}

Database

In this paper, ECG data is taken from Physionet: MIT-BIH Arrhythmia database for normal and VEBs. Total 678 normal and 81 VEBs taken from 10 records $(105,109,118,119$, 200, 202, 210, 214, 221 and 223).

Processing

Here, we applied, high pass filter with cutoff frequency $0.5 \mathrm{~Hz}$ to remove the baseline drift present in ECG signal. As the ECG datasets were having only high frequency (HF) noise, we used a low pass filter with cutoff frequency of $30 \mathrm{~Hz}$ to remove $\mathrm{HF}$ noise. Next, the Pan-Tompkins algorithm was applied to detect the R-peaks and difference operation method [13] was applied to find $\mathrm{Q}$ and $\mathrm{S}$ points on ECG beats.

\section{Feature extraction}

The following features were extracted from each beat of ECG to distinguish the VEBs from normal beats.

Table 1. Features extracted based on beat intervals

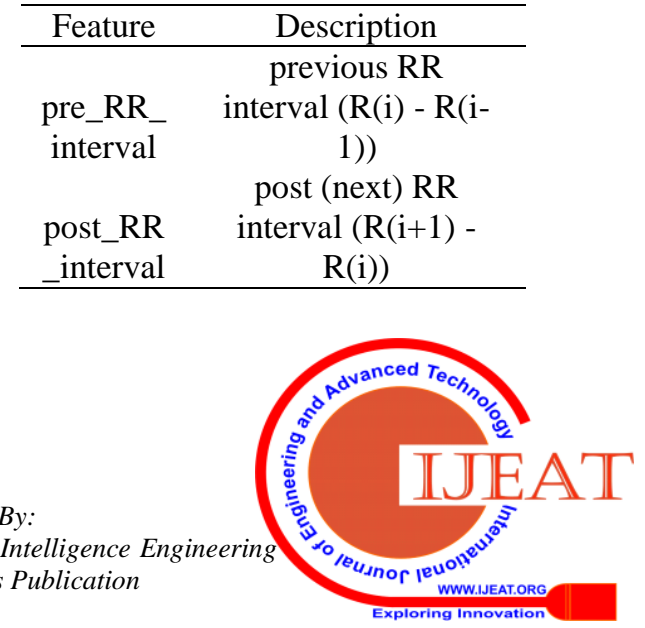




\section{Effect of PCA Feature Reduction on Ventricular Ectopic Beat Classification}

\begin{tabular}{cc}
\hline QRS & Time is taken for \\
duration & QRS complex \\
QR_slop & the slope between \\
e & Q and R points \\
RS_slop & the slope between R \\
e & $-\quad$ and S points \\
\hline
\end{tabular}

\subsection{Feature Reduction}

In this paper, we used Principal component analysis (PCA) features reduction technique [14] for testing the effect of FR on classification accuracy. Principal component analysis method projects a set of points on a smaller dimension subspace of best fit. It generates a new set of variables, called principal components. Each principal component is a linear combination of the initial variables. All the principal components are orthogonal to every different component, thus there is no redundant information.

\subsection{Classifter}

To verify the effect of FR on classification accuracy of VEBs, here we have applied 23 different classifiers. The list of classifiers is given in Table 2.

\section{RESULTS}

The ECG datasets taken from MIT-BIH Arrhythmia database are applied to Pan-Tompkins QRS detection algorithm to find R-peaks. Next, the Q, R, S points are found by a difference operation method. Therefore, the features: Pre- RR interval, post-RR interval, QRS duration, QR slope, and RS slope were cal- culated. The 5 features of 678 normal and 81 VEBs were applied to 23 different classifiers. PCA-FR reduced the dimension of the feature set from 5 to 3 (re- duction ratio [14] is 0.6). The beat detection rate without feature reduction and with PCA-FR are as shown in Table 3. The accuracy, Sensitivity, Specificity and Positive predictivity of all classifiers without and with FR are calculated by equations(1)-(4) and shown in Table 4 respectively.

$$
\begin{gathered}
\text { Sensitivity }(S e)=\frac{T P}{T P+F N} \\
\text { Specificity }(S p)=\frac{T N}{T N+F P} \\
P \text { ositiveP redictivity }(P p)=\frac{T P}{T P+F P}
\end{gathered}
$$

Table 2. List of different classifiers applied in this paper

$$
\begin{aligned}
& \text { model classifier Description } \\
& 1.1 \quad \text { fine tree A decision tree with many leaves with max } 100 \text { fine decisions } \\
& 1.2 \text { medium tree A decision tree of medium flexibility with fewer leaves and } \max 20 \\
& \text { decisions } \\
& \text { coarse tree A decision tree of few leaves and max } 4 \text { decisions } \\
& \text { fine KNN Number of nearest neighborhoods }(\mathrm{k})=1 \\
& \text { medium KNN Number of nearest neighborhoods }(\mathrm{k})=10 \\
& \text { coarse KNN Number of nearest neighborhoods }(\mathrm{k})=100 \\
& \text { cosine KNN the cosine Distance metric is used } \\
& \text { cubic KNN The cubic distance metric is used } \\
& \text { weighted KNN The weighted distance metric is used } \\
& \text { Linear SVM Linear separation using the linear kernel } \\
& \text { Quadratic SVM Linear separation using quadratic kernel } \\
& \text { Cubic SVM Linear separation using cubic kernel } \\
& \text { Fine Gaussian Linear separation using Gaussian kernel with scale s̄qrt (\# classes) / } 4 \\
& \text { medium Gaussian SVM Linear separation using Gaussian kernel with scalēsqrt (\# classes) } \\
& \text { Coarse Gaussian SVM Linear separation using Gaussian kernel with scale sqrt (\# classes)4 } \\
& \text { Boosted trees A decision tree with AdaBoost algorithm } \\
& \text { Bagged trees A boosted-aggregated ensemble of the fine decision tree } \\
& \text { subspace discriminant Discriminant classifier using random subspace algorithm } \\
& \text { subspace KNN KNN classifier using random subspace algorithm } \\
& \text { RUSBoosted trees A classifier with skewed data } \\
& \begin{array}{l}
\text { 4.1 Linear Discriminant Creates linear boundaries between classes } \\
\text { 4.2 Quadratic Discriminant Creates elliptic, parabolic and hyperbolic boundaries }
\end{array} \\
& 5 \text { Logistic Regression The function of a linear combination of predictors }
\end{aligned}
$$

$$
\text { ClassificationAccuracy }=\frac{T P+T N}{T P+F P+T N+F N}
$$


Table 3. Beats detection

\begin{tabular}{|c|c|c|c|c|c|}
\hline \multicolumn{2}{|c|}{ model classifier } & \multicolumn{4}{|c|}{ Before FR After FR TP } \\
\hline 1.1 & fine tree & 6601815 & 66 & 6542433 & 48 \\
\hline 1.2 & medium tree & 6601815 & 66 & 6542433 & 48 \\
\hline 1.3 & coarse tree & 6532525 & 56 & 6611742 & 39 \\
\hline 1.4 & fine KNN & 667119 & 72 & 6542423 & 58 \\
\hline 1.5 & medium KNN & 6611715 & 66 & 6562219 & 62 \\
\hline 1.6 & coarse KNN & 678081 & $\mathrm{O}$ & 678081 & $\mathrm{O}$ \\
\hline 1.7 & cosine KNN & 6522620 & 61 & 6512724 & 57 \\
\hline 1.8 & cubic KNN & 6601816 & 65 & 6562218 & 63 \\
\hline 1.9 & weighted KNN & 6651313 & 68 & 6582020 & 61 \\
\hline 2.1 & Linear SVM & 678081 & $\mathrm{O}$ & 678081 & $\mathrm{O}$ \\
\hline 2.2 & Quadratic SVM & 6717 & 68 & 6631524 & 57 \\
\hline 2.3 & Cubic SVM & 667119 & 72 & 6601822 & 59 \\
\hline 2.4 & Fine Gaussian SVM & 676236 & 45 & 6641425 & 56 \\
\hline 2.5 & $\begin{array}{l}\text { medium Gaussian } \\
\text { SVM }\end{array}$ & 6661216 & 65 & 6641426 & 55 \\
\hline 2.6 & $\begin{array}{l}\text { Coarse Gaussian } \\
\text { SVM }\end{array}$ & 6771 & 13 & 6780 & 1 \\
\hline 3.1 & Boosted trees & 6681015 & 66 & 6582026 & 55 \\
\hline 3.2 & Bagged trees & 6621611 & 70 & 6621624 & 57 \\
\hline 3.3 & $\begin{array}{l}\text { subspace } \\
\text { discriminant }\end{array}$ & 678081 & $\mathrm{O}$ & $6780 \quad 81$ & $\mathrm{O}$ \\
\hline 3.4 & subspace KNN & 6641429 & $\begin{array}{r}52 \\
75\end{array}$ & 6621636 & 45 \\
\hline 3.5 & RUSBoosted trees & 652266 & 75 & 6384011 & 70 \\
\hline 4.1 & Linear Discriminant & 673569 & 12 & 675370 & 11 \\
\hline 4.2 & $\begin{array}{l}\text { Quadratic } \\
\text { Discriminant }\end{array}$ & 6681026 & 55 & 6611739 & 42 \\
\hline 5 & Logistic Regression & 6780 & 4 & $67711 \quad 77$ & 4 \\
\hline
\end{tabular}

The scatter plot \& $\widetilde{\text { Receiver }} \widetilde{c}$ Operating Characteristics deriving many features from the signal, we tested the effect (ROC) of fine KNN classifier are shown in Figure $2 \& 3$ respectively. Finally, the effect of PCA-FR on the accuracy of all classifiers are shown in Figure 4.

\section{CONCLUSION}

As many classification systems involved the feature reduction concept for reducing the feature vector size after of feature reduction using PCA and presented in this paper. MIT-BIH Arrhythmia datasets were used for VEB classification by extracting Pre-RR interval, post-RR interval, QRS duration, QR slope, and RS slope. In this paper, the accuracy of VEB classification is tested with 23 different classifiers. It is observed that maximum classification accuracy of $97.4 \%$

\section{Table 4. Classification Performance}

\begin{tabular}{|c|c|c|c|c|}
\hline \multirow{2}{*}{\multicolumn{2}{|c|}{ model classifier }} & Before FR & \multicolumn{2}{|l|}{ After FR } \\
\hline & & Acc sp & Acc sp & PP \\
\hline 1.1 & fine tree & $95.781 .48 \% 97.35 \%$ & $97.78 \% 92.559 .26 \% 96.46 \%$ & $95.20 \%$ \\
\hline 1.2 & medium tree & $95.781 .48 \% 97.35 \%$ & $97.78 \% 92.559 .26 \% 96.46 \%$ & $95.20 \%$ \\
\hline 1.3 & coarse tree & $93.469 .14 \% 96.31 \%$ & $96.31 \% 92.248 .15 \% 97.49 \%$ & $94.03 \%$ \\
\hline 1.4 & fine $\mathrm{KNN}$ & $97.488 .89 \% 98.38 \%$ & $98.67 \% 93.871 .60 \% 96.46 \%$ & $96.60 \%$ \\
\hline 1.5 & medium KNN & $95.881 .48 \% 97.49 \%$ & $97.78 \% 94.676 .54 \% 96.76 \%$ & $97.19 \%$ \\
\hline 1.6 & se KNN & $89.30 .00 \% 100.00 \%$ & $89.33 \% 89.30 .00 \% 100.00 \%$ & $89.33 \%$ \\
\hline 1.7 & cosine $\mathrm{KNN}$ & $93.975 .31 \% 96.17 \%$ & $97.02 \% 93.370 .37 \% 96.02 \%$ & $96.44 \%$ \\
\hline 1.8 & cubi & 95.580 .2 & $97.63 \% 94.777 .78 \% 96$ & \\
\hline 1.9 & $\mathrm{KNN}$ & 96.683 .9 & $98.08 \% 94.775 .3$ & $97.05 \%$ \\
\hline 2.1 & r SVM & $9.30 .00 \% 100$ & $\% 89.30 .00 \% 100$. & 89.3 \\
\hline 2.2 & Quadratic SVM & $97.483 .95 \% 98.97 \%$ & $98.10 \% 94.970 .37 \% 97.79 \%$ & $96.51 \%$ \\
\hline 2.3 & Cubic SVM & $97.488 .89 \% 98.38 \%$ & $98.67 \% 94.772 .84 \% 97.35 \%$ & $96.77 \%$ \\
\hline 2.4 & Fine Gaussian SVM & $95.055 .56 \% 99.71 \%$ & $94.94 \% 94.969 .14 \% 97.94 \%$ & $96.37 \%$ \\
\hline 2.5 & medium Gaussian SVM & $96.380 .25 \% 98.23 \%$ & $97.65 \% 94.767 .90 \% 97.94 \%$ & $96.23 \%$ \\
\hline 2.6 & Coarse Gaussian SVM & $90.916 .05 \% 99.85 \%$ & $90.87 \% 89.51 .23 \% 100.00 \%$ & $89.45 \%$ \\
\hline 3.1 & Boosted trees & $96.781 .48 \% 98.53 \%$ & $97.80 \% 93.967 .90 \% 97.05 \%$ & $96.20 \%$ \\
\hline 3.2 & Bagged trees & $96.486 .42 \% 97.64 \%$ & $98.37 \% 94.770 .37 \% 97.64 \%$ & $96.50 \%$ \\
\hline 3.3 & subspace discriminant & $89.300 .00 \% 100.00 \%$ & $89.33 \% 89.30 .00 \% 100.00 \%$ & $89.33 \%$ \\
\hline 3.4 & subspace $\mathrm{KNN}$ & $94.364 .20 \% 97.94 \%$ & $95.82 \% 93.155 .56 \% 97.64 \%$ & $94.84 \%$ \\
\hline 3.5 & RUSBoosted trees & $95.892 .59 \% 96.17 \%$ & $99.09 \% 93.386 .42 \% 94.10 \%$ & $98.31 \%$ \\
\hline 4.1 & Linear Discriminant & $90.314 .81 \% 99.26 \%$ & $90.70 \% 90.413 .58 \% 99.56 \%$ & $90.60 \%$ \\
\hline 4.2 & Quadratic Discriminant & $95.367 .90 \% 98.53 \%$ & $96.25 \% 92.651 .85 \% 97.49 \%$ & $94.43 \%$ \\
\hline 5 & Logistic Regression & $89.94 .94 \% 100.00 \%$ & $89.80 \% 89.74 .94 \% \quad 99.85 \%$ & $89.79 \%$ \\
\hline
\end{tabular}




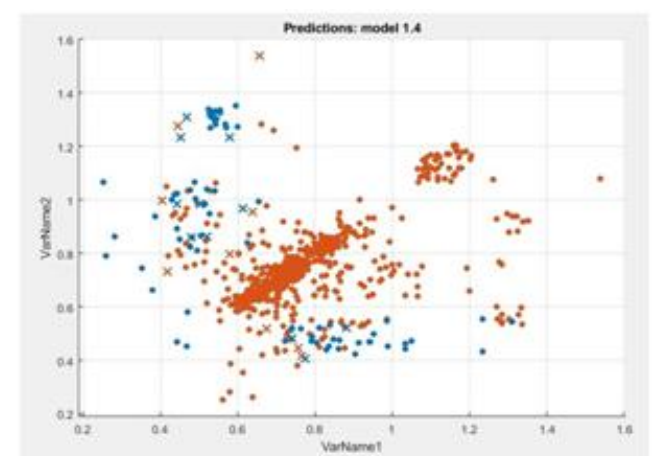

Fig. 2. Scatter Plot for Fine KNN classifier

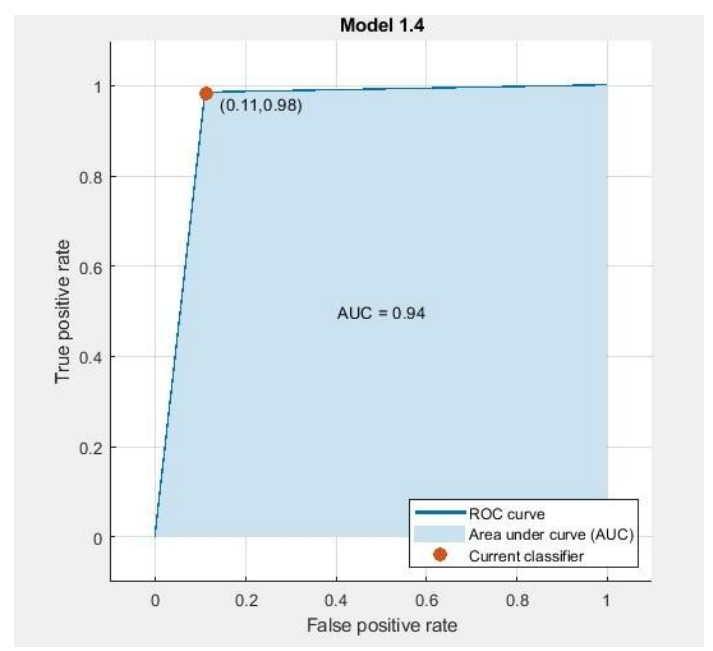

Fig. 3. Receiver Operating Characteristics (ROC) of Fine KNN classifier

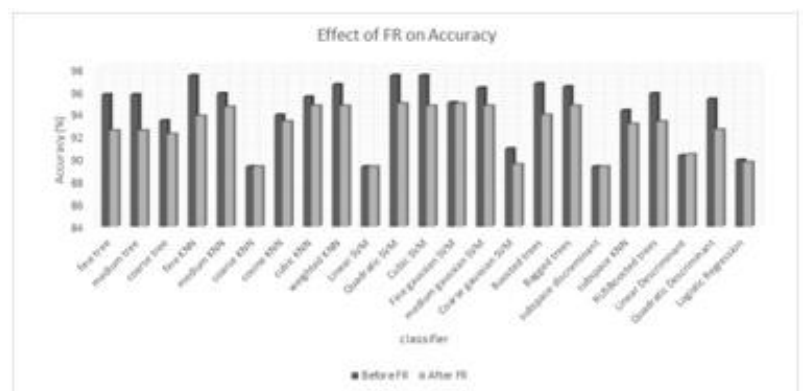

Fig. 4. Effect of PCA-FR on the accuracy of different classifiers

was achieved by fine KNN classifier and the accuracy was reduced by maximum $3.7 \%$ after applying PCA-FR. Therefore, it is concluded that the effect of PCA feature reduction on VEB classification accuracy is less significant, but it reduces the computational time when the feature vector size is very large.

\section{REFERENCES}

1. Ng, G. André,: Treating patients with ventricular ectopic beats. Heart 92.11, pp 1707-1712 (2006)

2. www.cardionetics.com/ventricular-ectopic-beats

3. James E Keany, et.al.,: Premature Ventricular Contraction. Medscape, Article, 761148, Updated: Jan 13 (2017)

4. [De Chazal, Philip: Detection of supraventricular and ventricular ectopic beats using a single lead ECG. In Engineering in Medicine and Biology Society (EMBC), 2013 35th Annual International Conference of the IEEE, IEEE, pp. 45-48 (2013)

5. De Chazal, Philip, et.al.: Automatic classification of heartbeats using ECG mor- phology and heartbeat interval features. IEEE
Transactions on Biomedical Engi- neering 51.7, pp. 1196-1206 (2004)

6. Sadiq, Ismail, and Shoab Ahmad Khan.: Heartbeat classification of ECGs us- ing morphology and beat intervals. In Bioinformatics and Biomedical Engineer- ing,(iCBBE) 2011 5th International Conference on, IEEE, pp. 1-5 (2011)

7. Hammed, Norhan S., and Mohamed I. Owis.: Patient adaptable ventricular ar- rhythmia classifier using template matching. In Biomedical Circuits and Systems Conference (BioCAS), IEEE, pp. 1-4 (2015)

8. Chow, H. S., Moody, G. B., \& Mark, R. G.: Detection of ventricular ectopic beats using neural networks. In Computers in Cardiology, Proceedings of IEEE, pp. 659- 662 (1992)

9. . Wiens, J., and John V. Guttag.: Patient-adaptive ectopic beat classification using active learning. In Computing in Cardiology, IEEE, pp. 109-112(2010)

10. Dotsinsky, Ivan A., and Todor V. Stoyanov.: Ventricular beat detection in single channel electrocardiograms. Biomedical engineering online 3 , no. 1 , pp. 3 (2004)

11. . Wiens, Jenna, and John V. Guttag.: Patient-specific ventricular beat classifica- tion without patient-specific expert knowledge: A transfer learning approach. In Engineering in Medicine and Biology Society, EMBC, 2011 Annual International Conference of the IEEE, pp. 5876-5879 (2011)

12. Baali, Hamza, and Mostefa Mesbah.: Ventricular ectopic beats classification using sparse representation and Gini Index. In Engineering in Medicine and Biology Society (EMBC), 2015 37 th Annual International Conference of the IEEE, pp. 5821$5824(2015)$

13. Yeh Yun-Chi, et.al.: QRS complexes detection for ECG signal: The Difference Operation Method. Computer methods and programs in biomedicine 91.3, pp 245- 254 (2008)

14. Vasan, et.al.: Dimensionality reduction using Principal Component Analysis for network intrusion detection. Perspectives in Science 8, pp 510-512. (2016) 